\title{
Tamoxifen Increases Membrane Fluidity at High Concentrations
}

\author{
Feride Severcan, ${ }^{1,4}$ Nadide Kazanci, ${ }^{2}$ and Faruk Zorlu ${ }^{3}$
}

Received March 30, 2000

\begin{abstract}
There are contradictory results in the literature relating to the effect of tamoxifen on membrane fluidity. The present work investigates the effect of tamoxifen on membrane dynamics to find out whether the concentration of tamoxifen can be one of the factors in this discrepancy. Turbidity (absorbance at $440 \mathrm{~nm}$ ) and Fourier transform infrared spectroscopic studies reveal that tamoxifen causes opposite effects on membrane fluidity at low (1 mol.\%) and high (30 mol.\%) tamoxifen concentrations. Low tamoxifen concentrations increase the absorbance in the gel and liquid crystalline phase, whereas high tamoxifen concentrations decrease the absorbance in gel and liquid crystalline phase, whereas tamoxifen concentrations decrease the absorbance. Observations on both phases show that the bandwidth of the $\mathrm{CH}_{2}$ stretching bands decreases with 1 mol.\% tamoxifen and increases with $30 \mathrm{~mol} . \%$ tamoxifen present, indicating a decrease in membrane fluidity at low tamoxifen concentrations and an increase in fluidity at high tamoxifen concentrations. It is seen that the apparent discrepancy in the literature on the effect of tamoxifen on membrane fluidity mainly arises from the tamoxifen concentration used and the confusion on the concept of lipid fluidity and lipid order.
\end{abstract}

KEYWORDS: Tamoxifen; model membrane; phospholipid membrane; FTIR; turbidity; membrane fluidity; membrane order.

\section{INTRODUCTION}

Tamoxifen is the trans geometric isomer of a substituted riphenylethylene. It has been used in the treatment of breast cancer since 1970, and is frequently used in the adjuvant treatment of women with breast cancer. It is also used for treating estrogen receptor positive patients with metastatic breast cancer. Post-menopausal, estrogen receptor-negative breast cancer patients can also benefit from adjuvant tamoxifen therapy, and some of the estrogen receptor-independent responses of cancer cells to tamoxifen may therefore be attributed to interactions of tamoxifen with cellular membranes [1, 2]. High dose tamoxifen has been used in the treatment of intracranial malignant gliomas and recurrent high grade gliomas. It has also been used in combination with chemotherapy in the treatment of metastatic malignant melanomas

\footnotetext{
${ }^{1}$ Department of Biology, Middle East Technical University, 06531, Ankara, Turkey.

${ }^{2}$ Department of Physics, Faculty of Science, Ege University, 35100, Izmir, Turkey.

${ }^{3}$ Department of Radiation Oncology, Medical School, Hacettepe University, Ankara, Turkey.

${ }^{4}$ To whom correspondence should be addressed: E-mail: feride@metu.edu.tr
} 
[1,2]. It acts like estrogen in some tissues, but behaves like estrogen blockers in others. Estrogenic properties of tamoxifen cause beneficial as well as harmful side effects. Beneficial estrogenic effects from tamoxifen include a decrease in total cholesterol and inhibition of osteoporosis in post menopausal women [3,4]. Tamoxifen and derivatives have been shown to protect biological membranes, as well as membrane-model systems such as liposomes, against free radical mediated lipid peroxidation. Observation of this protective action of tamoxifen and its derivatives can be attributed to molecular modulation of the membrane environment by the interaction of sterols with the particular fatty acid side-chains present in membrane phospholipids [5].

In common with most anticancer agents, tamoxifen is a highly lipophilic, amphiphatic molecule, and therefore it may accumulate in the lipid part of the membrane [6]. It is suggested that most of these drugs cause their toxic effects through incorporation into cell membranes. Studies of the interactions of the drugs with biomembranes are important in understanding the mechanism of their action.

The effect of tamoxifen on model and natural membranes has been investigated previously by Fourier transform infrared (FTIR) and fluorescence spectroscopy [6-13]. These studies reported a tamoxifen-induced disordering effect (increase in chain flexibility) in membranes [6,11-13]. On the other hand, there are conflicting results on the effect of tamoxifen on membrane dynamics. Most of the studies reported the stabilizing effect of it on membrane, by restricting the mobility of the lipids [6-10]. However, an increase in membrane fluidity modulated by tamoxifen was recently reported $[12,13]$. It is generally observed that tumor cells become resistant to the drug with which they were initially treated. The reason for this phenomenon is still unknown. One of the suggestions for this is the alteration in the dynamic properties of the cell membrane [14]. For this reason, it is important to have better understanding of the effect of tamoxifen on membrane fluidity for the determination of the proper dose to be used in the treatment. In our previous FTIR study we showed that the addition of tamoxifen, up to $20 \mathrm{~mol} \%$, into DPPC liposomes disorders the lipid acyl chains while, at the same time, decreases membrane dynamics [6]. The present Fourier transform infrared (FTIR) spectroscopic study and turbidity measurements at $440 \mathrm{~nm}$ have been performed to understand whether higher concentrations of tamoxifen exert different effects on membrane dynamics than do lower concentrations. Such a difference may be one of the reasons for discrepancy reports in the literature on tamoxifen modulated membrane fluidity.

\section{MATERIALS AND METHODS}

Tamoxifen and DPPC were purchased from Sigma (St. Louise, Mo).

For turbidity studies pure phospholipid multilamellar liposomes were prepared according to the procedure reported in Severcan et al. [15]. Residual solvent was removed by subjecting the films to vacuum drying by spin-vacuum (HETO-spin vac) system for two hours. Dry thin film was hydrated by $10 \mathrm{mM}$ phosphate buffer ( $\mathrm{pH}$ 7.4). Multilamellar liposomes were formed by vortexing the mixture for $20 \mathrm{~min}$. at a temperature about $20^{\circ} \mathrm{C}$ above the main phase transition temperature of the lipid. Tamoxifen-containing liposomes were prepared by exactly the same procedure 
but in this case, at the beginning of the sample preparation the desired amount of tamoxifen dissolved in ethanol was first put into a round bottomed flask, excess of organic solvent was evaporated by nitrogen flux and then phospholipid was added. Turbidity studies were carried out in a Shimadzu A160 double beam spectrophotometer. The details were reported in Severcan et al. [15]. Turbidity studies are considered as a special form of light scattering experiments. Scattering is usually detected by measuring the intensity of radiation at some angle $\theta$ to the incident wave, but it may also be detected by the reduction in the transmitted light with $\theta=$ 0 . The latter is called turbidity [16].

The lipid mixture for infrared measurements was prepared by a procedure similar to the procedure reported in Severcan et al. [15]. Infrared spectra were obtained using a BOMEM, MB-157 FTIR spectrometer. $20 \mu$ l of sample suspension was placed between $\mathrm{CaF}_{2}$ windows, separated by a $12-\mu \mathrm{m}$ mylar spacer. Interferograms were averaged for 100 scans at $2 \mathrm{~cm}^{-1}$ resolution. The temperature was regulated by a Unicam Specac digital temperature controller unit and a thermocouple located on the edge of the cell window. The samples were incubated for $10 \mathrm{~min}$ at each temperature before spectral scanning.

\section{RESULTS AND DISCUSSION}

Turbidity studies have been performed at 440 and $550 \mathrm{~nm}$. These wavelength values have been chosen to minimize light scattering [17]. The technique has been previously used in model and biological membrane studies to monitor the membrane phase transition behavior [15, 17-22].

Figure 1 shows the temperature dependence of the absorbance values at $440 \mathrm{~nm}$ for DPPC liposomes in the absence and presence of low $(1 \mathrm{~mol} . \%)$ and high (30 mol.\%) concentrations of tamoxifen. As seen from the figure, the absorbance decreases for pure DPPC liposomes as the system goes from the gel to the liquid crystalline phase. This can be explained by the change in turbidity that accompanies the gel to liquid crystalline phase transition, due to the change in refractive index as the lipid density changes during melting [18]. The well-known phase transition observed at $41.5^{\circ} \mathrm{C}$ is consistent with previous turbidity studies of DPPC membranes $[19,21]$. With the addition of 1 mol.\% tamoxifen, the absorbance slightly increases, however, the absorbance dramatically decreases in the presence of $30 \mathrm{~mol} . \%$ tamoxifen. Similar behavior has been obtained for turbidity studies at $550 \mathrm{~nm}$ (not shown). The decrease in absorbance with the addition of high concentration tamoxifen can either reflect a decrease in aggregation and fusion (decrease in liposome size) [22], or most probably indicates an increase in lipid mobility due to the change in refractive index of the medium $[17,18]$.

To asertain the opposite effect of tamoxifen at low and high concentration, we carried out an FTIR study and investigated the $\mathrm{CH}_{2}$ stretching bands. The bandwidth of the $\mathrm{CH}_{2}$ antisymmetric and symmetric stretching vibration of the lipid acyl chain, which appears around $2920 \mathrm{~cm}^{-1}$ and $2850 \mathrm{~cm}^{-1}$ respectively, reflects information about the dynamics of the membrane [23, 24]. 


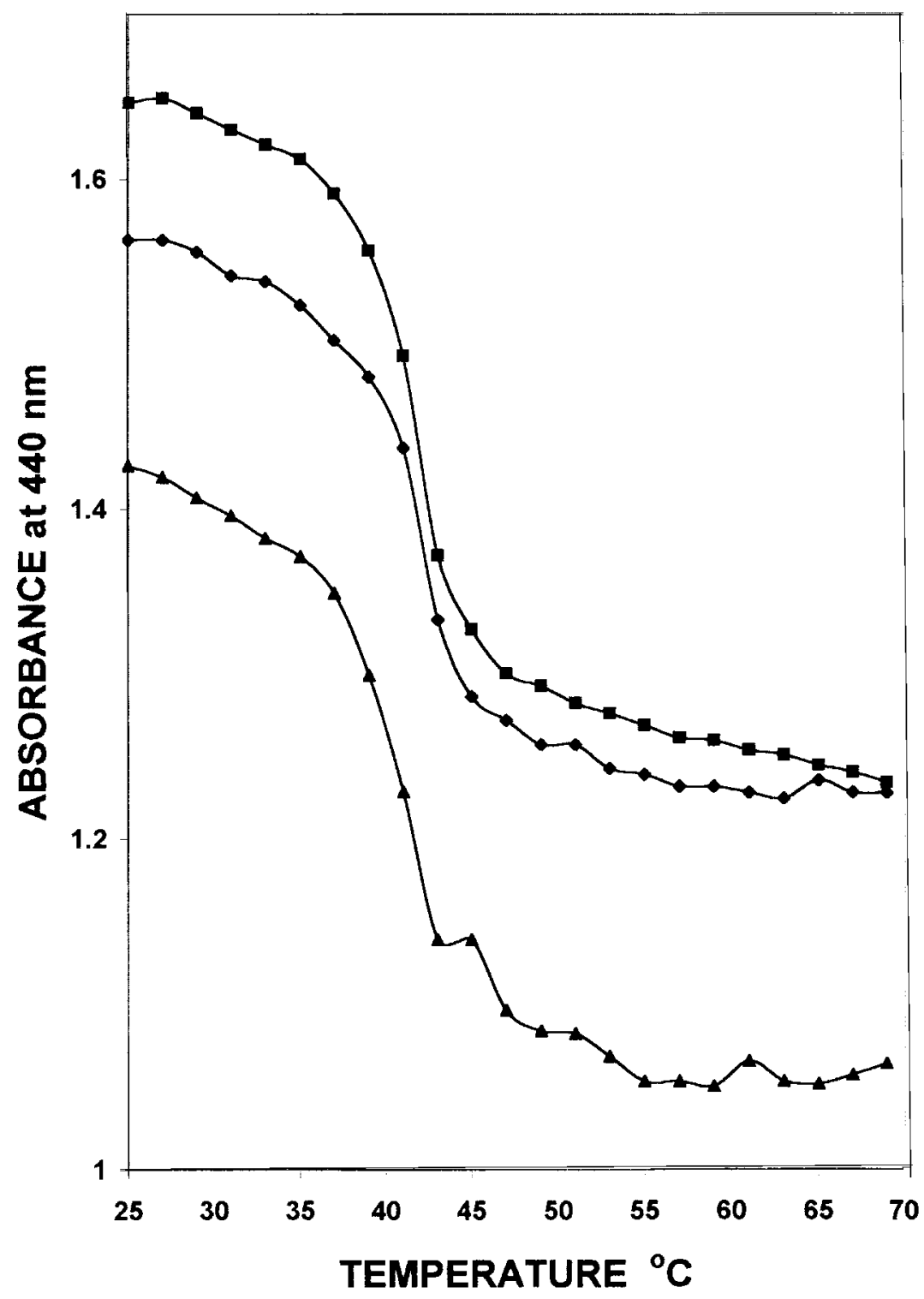

Fig. 1. Temperature dependence of absorbance at $440 \mathrm{~nm}$ for DPPC liposomes in the presence of $(\bullet) 0$ mol.\% TAM, (○) 1 mol.\% TAM, (A) 30 mol.\% TAM.

Figure 2 shows the temperature dependence of the bandwidth of the $\mathrm{CH}_{2}$ antisymmetric stretching bands in the infrared spectra of DPPC liposomes in the presence and absence of low $(1 \mathrm{~mol} \%$ ) and high (30 mol.\%) concentration of tamoxifen. With the addition of 1 mol.\% tamoxifen into the DPPC membranes, the bandwidth decreases both in the gel and liquid crystalline phase. By contrast, the 


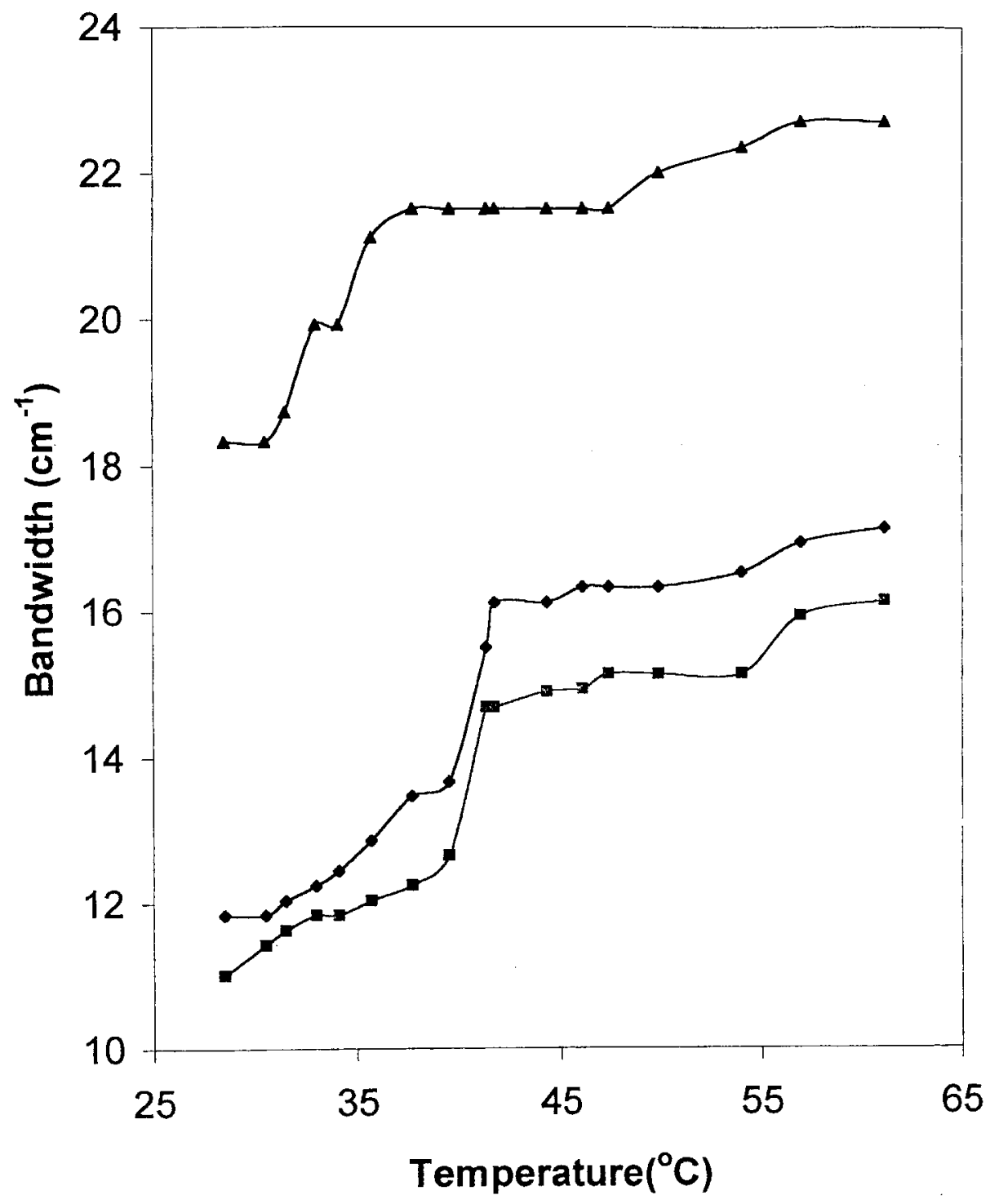

Fig. 2. The temperature dependence of the bandwidth of the $\mathrm{CH}_{2}$ antisymmetric stretching bands in the infrared spectra of DPPC liposomes in the presence of $(\bullet) 0 \mathrm{~mol} . \% \mathrm{TAM},(\boldsymbol{\square}) 1 \mathrm{~mol} . \%$ TAM, (А) 30 mol.\% TAM (the bandwidth was measured at $0.75 \times$ peak-height position).

bandwidth dramatically increases in both phases in the presence of high tamoxifen concentration. Similar behavior has also been observed for the $\mathrm{CH}_{2}$ symmetric stretching bands (not shown).

The peak frequencies of the $\mathrm{CH}_{2}$ stretching bands have been examined to see whether tamoxifen also affects membrane order. The introduction of gauche conformers produces shifts in the $\mathrm{CH}_{2}$ stretching bands to higher frequencies [24, 25]. 


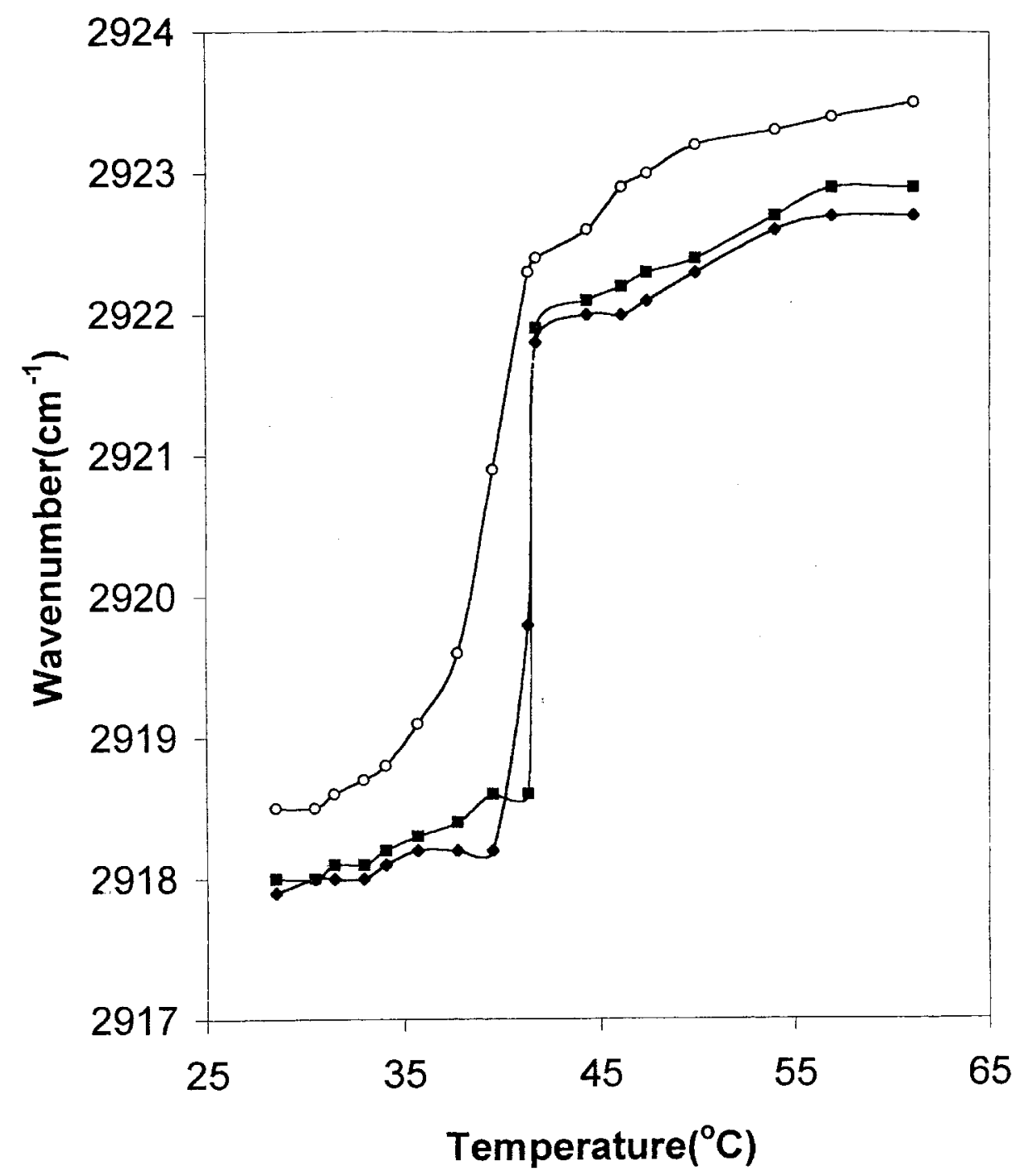

Fig. 3. The temperature dependence of the peak frequence of the $\mathrm{CH}_{2}$ antisymmetric stretching bands in the infrared spectra of DPPC liposomes in the presence of $(\checkmark) 0$ mol.\% TAM, 1 mol.\% TAM, (O) 30 mol.\% TAM.

Figure 3 shows the temperature dependence of the frequency of the $\mathrm{CH}_{2}$ antisymmetric stretching bands in the infrared spectra of DPPC liposomes in the presence and absence of low $(1 \mathrm{~mol} . \%)$ and high $(30 \mathrm{~mol} . \%)$ concentration of tamoxifen. As seen from the figure, for pure DPPC liposomes, the system shows two transitions. A minor transition at $35^{\circ} \mathrm{C}$ and a more abrupt transition around $41^{\circ} \mathrm{C}$ which correspond to the pre $\left(T_{\mathrm{p}}\right)$ and main phase transition $\left(T_{\mathrm{m}}\right)$ of DPPC, respectively. With the addition of 1 mol.\% tamoxifen into the DPPC membranes, a similar phase transition curve was observed. The main phase transition shifted to a somewhat lower 
temperature and the frequency of the $\mathrm{CH}_{2}$ stretching bands slightly increased in the gel and in the liquid crystalline phase. Addition of $30 \mathrm{~mol} . \%$ tamoxifen into the DPPC system broadens the phase transition curve, shifted the main transition to lower temperatures and increased the frequency in both phases. The broadening in the curve implies a loss of cooperativity between the lipid chains with the insertion of tamoxifen into the DPPC membrane. The increase in the frequency represents an increase in the number of gauche conformers indicating that tamoxifen disorders the membrane at high and low concentrations.

The results of present lipid turbidity and infrared study reveal that membrane fluidity decreases in the presence of $1 \mathrm{~mol} \%$ tamoxifen and increases in the presence of $30 \mathrm{~mol} . \%$ tamoxifen while a decrease in membrane order is observed for both concentrations. These results have been observed in both the gel and the liquid crystalline phase. The results for low tamoxifen concentration are in agreement with our previous FTIR study on DPPC membranes where we varied the tamoxifen concentration between 3-20 mol.\% [6]. Wiseman et al. also reported a tamoxifen-modulated decrease in membrane fluidity as measured with DPH fluorescence anisotropy $[8,9]$ where low concentrations of tamoxifen were used. Dicko et al. recently showed that the peak frequency of the $\mathrm{CD}_{2}$ stretching bands of DMPC liposomes in the FTIR spectra, increases for tamoxifen molar ratio of $25 \%$ [13] which is in agreement with our previous [6] and present findings. However, in that study this disordering effect(increase in the $\mathrm{CD}_{2}$ frequency) was interpreted as a fluidizing effect. These two terms, order and fluidity, should be used with care since fluidity refers to the rate of motion but not to the ordering of the molecular system and no simple relation between the two quantities is known [26]. In the same study fluidizing effect of tamoxifen on brain membranes was also reported as determined by fluorescence depolarization of the probe DPH by stating that their results contradicts with previous fluorescence studies [8,9]. In the study of Dicko et al. [13], the tamoxifen concentration used is close to the higher concentration used in our study. So it seems that there is no contradiction of this work with previous fluorescence studies $[8,9]$, instead the higher tamoxifen concentration used seems to cause the apparent discrepancy. In disagreement with previous data [6-10], Custodio et al. [11] reported a different effect of tamoxifen in the gel and the liquid crystalline phase. According to this work, tamoxifen stabilizes the membrane (for low concentration) in the liquid crystalline phase. Whilst a strong fluidizing effect is observed in the gel phase as measured by fluorescence polarization of DPH. Recently Luxo et al. [12] used the same technique to obtain information about the effect of tamoxifen at low concentrations, on membrane order and membrane order-disorder transition, interpreting the disordering effect as a fluidity increase. These studies reveal that, in addition to concentration effect of tamoxifen on membrane fluidity, the confusion between the concepts of membrane order and dynamics in the literature may be the reason for the apparent discrepancy of tamoxifen modulated membrane fluidity.

It is very interesting that tamoxifen destabilizes the membrane at high concentrations, opposite to its effect on membrane at low concentration. The precise mechanism of action for tamoxifen is uncertain, although many biological effects have been documented with its use. For this reason, further detailed temperature, composition and concentration dependent spectroscopic studies on tamoxifen-membrane 
interactions with different techniques are necessary to understand its action mechanism at molecular level. Understanding the effect of tamoxifen concentration will be helpful in determining the proper dose and will improve the effective use of it in the treatment of several deseases.

\section{ACKNOWLEDGEMENTS}

This work has been supported by State Planning Organization of Turkey (DPT98K112530/AFP98010805).

\section{REFERENCES}

1. Furr, B. J. A., and Jordan, V. C. (1984) Pharmacol. Ther. 25:127-205.

2. Wiseman, H., Laughton, M. J., Arnstein, H. R., Cannon, M., and Halliwell, B. (1990) FEBS Lett. 263:192-194.

3. Wiseman, H. (1994) Tamoxifen, John Wiley and Sons, pp. 31-59.

4. Love, R. R, Mazess, R. B., and Torney, D. C. (1988) Breast Cancer Res. Treat. 12:297-301.

5. Wiseman, H. (1994) Tamoxifen, John Wiley and Sons, pp. 118-159.

6. Boyar, H., and Severcan, F. (1997) J. Mol. Structure 408/409:265-268.

7. Wiseman, H. (1993) FEBS Lett. 326:285-288.

8. Wiseman, H., Cannon, M., Arnstein, H. R. V., and Halliwell, B. (1992) Biochim. Biophys. Acta 1138: $197-202$.

9. Wiseman, H., Quinn, P., and Halliwell, B. (1993) FEBS Lett. 330:53-56.

10. Wiseman, H., Cannon, M., Arnstein, H. R. V., and Halliwell, B. (1993) Biochim. Biophys. Acta 1181:201-206.

11. Custodio, J. B. A., Almeida, L. M., and Madeira, V. M. C. (1993) Biochim. Biophys. Acta 1150:123129.

12. Luxo, C., Jurado, A. S., and Madeira, V. M. C. (1998) Biochim. Biophys. Acta 1369:71-84.

13. Dicko, A., Morissette, M., Ameur, S. B., Pezolet, M., and Di Paolo, T. (1999) Brain Research Bulletin 49:401-504.

14. Ramu, A., Glaubiger, D., Magrath, I. T., and Joshi, A. (1983) Cancer Res, 43:5533-5537.

15. Severcan, F., Kazanci, N., Baykal, Ü., and Suzer, S. (1995) Biosci. Reps. 15:221-229.

16. Freifelder, D. (1982) Biophysical Chemistry, Freeman and Company, New York.

17. Chang, C. S., and Colbow, K. (1976) Biochim. Biophys. Acta 436:260-282.

18. Yi, P. N., and MacDonalt, R. C. (1973) Chem. Phys. Lipids 11:114-134.

19. Viti, V., Agostini, S., Ceccarini, M., and Mineti, M. (1985) Physio. Chem. Phys. Med. NMR 17:413420.

20. Stillwell, W., and Wassall, S. R. (1990) Methods in Enzymology 189: 373-382.

21. Eker, F., Durmus, H. O., Akinoglu, B. G., and Severcan, F. (1999) J. Mol. Structure 482/483:693697.

22. Ohki, S., and Düzgüne, N. (1979) Biochim. Biophys. Acta 552:438-449.

23. Casal, H. L., Cameron, I. C. P., Smith, I. C. P., and Mantsch, H. H. (1980) Biochemistry 1917:444451.

24. Cameron, P. G., and Chatette, G. N. (1981) Appl. Spectrosc. 35:224-229.

25. Hubner, W., Mantsch, H. H., and Kates, M. (1991) Biochim. Biophys. Acta 1066:166-174.

26. Seelig, A., and Seelig, J. (1974) Biochemistry 13:4839-4845. 